\title{
Characterization of cascaded forward Brillouin scattering seeded by backward stimulated Brillouin scattering in optical fibers
}

\author{
Neisei Hayashi ${ }^{1,2,3}$, Yosuke Mizuno ${ }^{2,4, \text { a) }}$, Heeyoung Lee ${ }^{5}$, Kentaro Nakamura $^{2}$, Sze Yun Set ${ }^{3}$ and Shinji Yamashita $^{3}$
}

Abstract We present a method for generating cascaded forward Brillouin scattering (CFBS) on the basis of a counter-propagated pump-probe technique with backward stimulated Brillouin scattering used as its seed. The CFBS, induced by forward stimulated Brillouin scattering (FSBS), is generated via the energy transfer from the probe light to other acoustic resonance frequencies. The CFBS spectrum generated in a 390-m-long highly nonlinear fiber exhibits a high signal-to-noise ratio (SNR), and the center frequencies of its acoustic resonance peaks agree with theoretical values. In addition, the SNR dependence on pump/probe powers and the CFBS frequency shift dependence on temperature are investigated.

Keywords: Brillouin scattering, optical fiber sensing, temperature sensing Classification: Optical hardware (fiber optics, microwave photonics, optical interconnects, photonic signal processing, photonic integration and modules, optical sensing, etc.)

\section{Introduction}

Forward Brillouin scattering (FBS) is a fiber-optic nonlinear optical phenomenon induced by the interaction between incident light and acoustic waves propagating through the cross-sectional area of the fiber $[1,2]$. The propagating direction of FBS is the same as that of the incident light. FBS has been observed in various kinds of fibers, such as silica single-mode fibers (SMF) [1], polarization-maintaining fibers [3], and few-mode fibers [4]. When stimulated using co-propagated pump and probe lights $[5,6]$, it is called forward stimulated Brillouin scattering (FSBS). In the FSBS process, if the pump power is higher than that of the probe light and the center frequency of the probe light is the same as that of the first acoustic resonance peak of FBS, the energy of the pump is transferred to other acoustic resonance frequencies. This phenomenon is called cascaded FBS (CFBS) [5, 7, 8]. The signal-to-noise ratio (SNR) of CFBS is reported to be higher than that of FBS [5].

${ }^{1}$ The Graduate School for the Creation of New Photonics Industries, Shizuoka 431-1202, Japan

2 Institute of Innovative Research, Tokyo Institute of Technology, Yokohama 226-8503, Japan

${ }^{3}$ Research Center for Advanced Science and Technology, The University of Tokyo, Tokyo 153-8904, Japan

${ }^{4}$ Faculty of Engineering, Yokohama National University, Yokohama 240-8501, Japan

${ }^{5}$ College of Engineering, Shibaura Institute of Technology, Tokyo 135-8548, Japan

a) mizuno-yosuke-rg@ynu.ac.jp

\section{DOI: $10.1587 /$ elex.17.20200139}

Received April 9, 2020

Accepted May 21, 2020

Publicized June 5, 2020

Copyedited June 25, 2020
To date, FBS has been used to develop megahertz-order digital logic devices [9], megahertz-order radio-frequency signal generators [10], temperature sensors [11], strain sensors [12], fiber-diameter sensors [13], and acousticimpedance sensors [14, 15, 16]. Above all, acousticimpedance sensing is a unique advantage of FBS-based sensors, with which the external acoustic impedance can be measured using only the linewidth of the FBS spectrum. If distributed acoustic-impedance sensing is achieved using conventional spatially resolving techniques $[17,18,19,20$, $21,22,23,24,25]$, it could be exploited to implement distributed discriminative multi-parameter sensors for cancer detection in the human body [26, 27], underground oil layer detection [28], quality inspection of bioethanol [29], $\mathrm{NaCl}$ concentration detection in water for cultivation of delicate tuna [30], etc. In addition, if transverse acoustic waves can be localized using a conventional correlation-domain technique in multi-core fibers, active distributed phase modulators [31] may be implemented. Note that distributed FBS sensing has been already demonstrated, but only on the basis of specially configured time-domain techniques [32, 33].

One of the major problems in achieving FBS-based distributed sensing is the propagating direction of the FBS, which prevents simple application of the conventional distributed Brillouin sensing techniques. To overcome this problem, in 2004, Tanaka et al. [34] proposed backward observation of FBS coupled with backward stimulated Brillouin scattering (BSBS), but the SNR of the backwardly observed FBS was extremely low because the Brillouin gain coefficient of FBS is much smaller than that of CFBS.

In this work, first, we propose a method for generating CFBS seeded by BSBS using a counter-propagated pumpprobe technique. In this method, the center frequency of the probe light is downshifted from that of the pump light by the sum of the Brillouin frequency shift (BFS) of BSBS and the first-order acoustic-resonance frequency shift of FBS. The CFBS induced by FSBS is generated via the energy transfer from the probe light to other acoustic resonance frequencies. Then, we present the results of experimental observation of the CFBS in a 390-m-long highly nonlinear fiber (HNLF) using BSBS as its seed. The measured CFBS has a high SNR and its center frequencies of the acoustic resonance peaks agree with theoretical values. Finally, we clarify the Brillouin properties of the generated CFBS peak at $941 \mathrm{MHz}$, such as the SNR dependence on pump and probe powers and the BFS dependence on temperature. As regards the SNR dependence on pump, the threshold of the CFBS is found 
to be $13.0 \mathrm{dBm}$ with a $-5.0-\mathrm{dBm}$ probe light. As for the SNR dependence on probe, the SNR of the CFBS is optimized with a $17.0-\mathrm{dBm}$ pump light and $-5.0-\mathrm{dBm}$ probe light. The temperature dependence coefficient of the BFS is measured to be $99 \mathrm{kHz} / \mathrm{K}$, which is close to the theoretical value. Note that this work complements the preliminary results presented in [35]. The additional value of this extended paper lies in providing further results of the CFBS spectrum dependencies on pump/probe power and temperature.

\section{Principle}

Various kinds of acoustic waves caused by thermal fluctuations exist in optical fibers [1]. One of these acoustic waves is the radial acoustic mode $\left(\mathrm{R}_{0, m}\right.$, where $m$ is the acoustic resonance mode), which literally induces displacements in the radial direction and also perturbs the refractive index in the fiber [1]. Optical scattering based on the $\mathrm{R}_{0, m}$ mode is referred to as polarized FBS. The central frequency of the $m$-th acoustic mode $\left(v_{\mathrm{GB}, m}\right)$ is given by

$$
v_{\mathrm{GB}, m}=\frac{v y_{m}}{\pi d},
$$

where $d$ is the fiber's outer diameter, $v$ is the velocity of the longitudinal acoustic waves, and $y_{m}$ is the value derived from the following equation [1]:

$$
\left(1-\alpha^{2}\right) J_{0}\left(y_{m}\right)-\alpha^{2} J_{2}\left(y_{m}\right)=0,
$$

where $\alpha$ is the shear acoustic velocity per longitudinal acoustic velocity, $J_{0}$ is the zero-order Bessel function, and $J_{2}$ is the second-order Bessel function. The central frequency is known to depend on temperature, with a dependence coefficient $(m=11)$ of $43.5 \mathrm{kHz} / \mathrm{K}$ in silica SMFs [36]. The coefficient of temperature dependence of the FBS spectrum is given by [11]:

$$
\frac{d v_{\mathrm{GB}, m}}{d T}=\left(\frac{1}{v} \frac{d v}{d T}\right) v_{\mathrm{GB}, m}=K v_{\mathrm{GB}, m},
$$

where $K$ is a constant $\left(8.6 \times 10^{-5} /{ }^{\circ} \mathrm{C}\right.$ in silica SMFs) [11]. The $K$ value in the HNLF appears to be almost the same as that in a silica SMF, because the core/cladding materials of the HNLF and an SMF are similar to each other $[6,11]$. When the pump light and the probe light with center frequency shifted by the amount of acoustic resonance frequency from the pump light are injected into an optical fiber, the energy of the pump is transferred to the probe light, which enhances the probe light. This phenomenon is called FSBS [6]. The pump power of FSBS is transferred to a higher Stokes order $[5,8]$ when the pump power is higher than that of the probe power and the center frequency of the probe light is congruent with that of the first acoustic resonance frequency. This cascading process is unique to

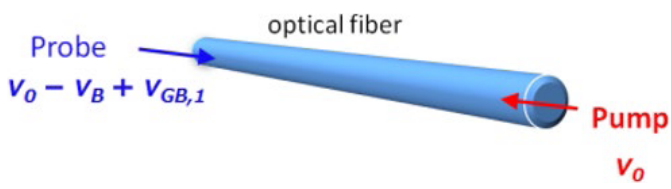

Fig. 1 Schematic configuration of pump-probe co-propagation for CFBS generation.
FBS, although the cascaded optical fields coherently drive the same phonon field producing successive parametric frequency shifts [5].
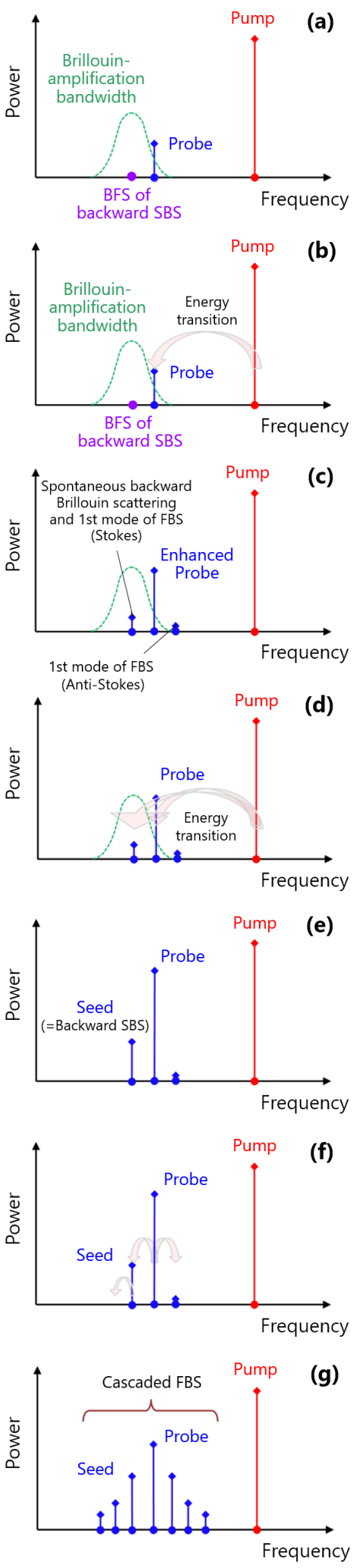

Fig. 2 Generation process of CFBS using BSBS as seed. (a) Initial state of pump and probe light. (b) Energy transfer from pump to probe light. (c) FBS generation by enhanced probe. (d) Enhancement of first resonance FBS peak and probe. (e) Generation of BSBS as seed of CFBS. (f) Energy transfer from probe to the seed. (g) Generation of CFBS using BSBS as seed. 
The basic concept underlying the principle of the CFBS generation seeded by BSBS is depicted in Figs. 1 and 2 . When we inject pump light with a center frequency of $v_{0}$ and probe light with a center frequency of $v_{0}-v_{B}+v_{G B, 1}$ (see Figs. 1 and 2(a)), the energy of the pump light is transferred to the probe light (see Fig. 2(b)) [37] because the BSBS linewidth is sufficiently broad. Then, FBS is generated by the enhanced probe (see Fig. 2(c)) [9], and the energy of the pump is further transferred to the acoustic resonance peak of the FBS and probe light (see Fig. 2(d)). Subsequently, this FBS signal and spontaneous backward Brillouin scattering generated by the pump light are enhanced by the pump light (see Fig. 2(e)) [5, 7], which generates BSBS for seeding CFBS (see Fig. 2(f)). Finally, the energy of the probe light is transferred to the seed and CFBS is generated (see Fig. 2(g)) [8].

\section{Experimental setup}

In experiment, we employed an ultraviolet curable coated 390-m-long silica HNLF (HNDS1614CA-4-3-3, Sumitomo) as a fiber under test (FUT). This HNLF had a core diameter of $3.5 \mu \mathrm{m}$, a cladding diameter of $117 \mu \mathrm{m}$, a core refractive index of $\sim 1.46$, a dispersion-shifted structure, and a propagation loss of $\sim 0.76 \mathrm{~dB} / \mathrm{km}$ at $1.55 \mu \mathrm{m}$. The BFS was $9.092 \mathrm{GHz}$ at $1.55 \mu \mathrm{m}$, and the Brillouin threshold power was $13 \mathrm{dBm}$ with the pump-probe technique. The schematic setup used to observe the CFBS spectrum (Fig. 3) is fundamentally the same as that previously reported [9]. All the optical paths excluding the FUT are standard silica SMFs. The output from a distributed-feedback laser diode at $1546 \mathrm{~nm}$ (linewidth: $1.2 \mathrm{kHz}$, output power: $<6 \mathrm{dBm}$ ) was divided into two: probe and pump lights. The center frequency of the probe light was shifted by several gigahertz with a single-sideband modulator (SSBM; carrier suppression ratio: $>20 \mathrm{~dB})$. This value well covers the BFS $\left(v_{B}\right)$ of backward SBS and the first acoustic resonance frequency of FBS. After power amplification using an erbium-doped fiber amplifier (EDFA), the probe light was injected into the FUT. The pump light was injected into the FUT from the other end, after amplified using another EDFA. The CFBS light was guided to a photodetector (PD) along with the transmitted probe light via a variable optical attenuator (VOA). Using the PD, the beat signal between the CFBS and the transmitted probe light was converted into an electrical signal, which was observed using an electrical spectrum analyzer (ESA). The polarization state in the FUT was averaged with a polarization scrambler (PSCR). The amplified spontaneous emission noise from each EDFA was suppressed using two optical filters (OF) with a narrow bandwidth $(\sim 10 \mathrm{GHz})$. The ambient temperature was $27^{\circ} \mathrm{C}$.

\section{Experimental results}

Figures 4(a)(b) show the CFBS spectrum observed for a pump power of $13.9 \mathrm{dBm}$ and a probe power of $-5.0 \mathrm{dBm}$. The attenuation of the VOA was set to $8.7 \mathrm{~dB}$. The horizontal axis was normalized by subtracting the electrical noise of the PD and the ESA as the noise floor from the mea-

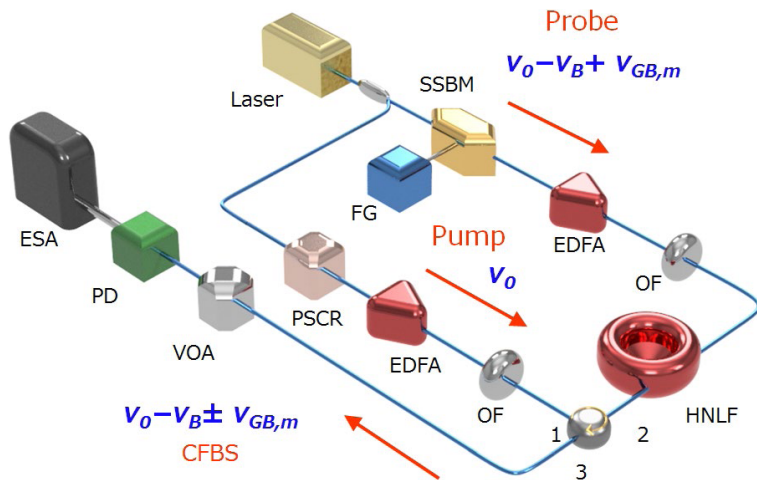

Fig. 3 Schematic setup used to observe the cascaded forward Brillouin scattering (CFBS) using backward stimulated Brillouin scattering (BSBS) as seed. EDFA: erbium-doped fiber amplifier, ESA: electrical spectrum analyzer, FG: function generator, HNLF: highly nonlinear fiber, OF: optical filter, PD: photodetector, PSCR: polarization scrambler, SSBM: singlesideband frequency modulator; VOA: variable optical attenuator.
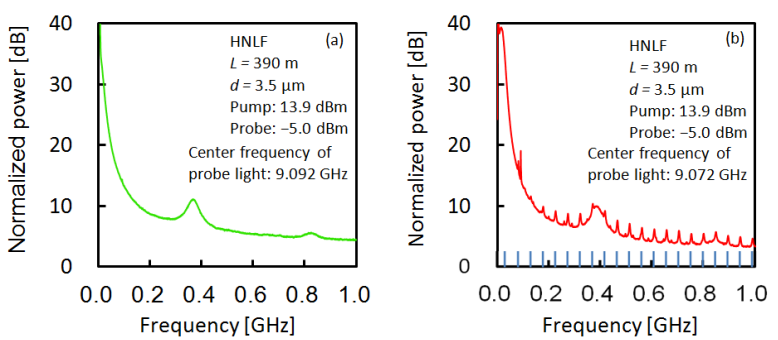

Fig. 4 (a) Wide-range view of the spectrum when the center frequency of the probe light was set to the Brillouin frequency shift (BFS). (b) Widerange view of the observed CFBS spectrum (red), and theoretical center frequencies of $R_{0, m}$ (blue).

sured spectrum. When the center frequency of the probe light was set to $9.092 \mathrm{GHz}$, a small peak was observed at $400 \mathrm{MHz}$, which originates from the second Brillouin peak (see Fig. 4(a)). Further, when the center frequency of the probe was set to $9.072 \mathrm{GHz}$, approximately 20 clear peaks appeared in the frequency range from $20 \mathrm{MHz}$ to $1000 \mathrm{MHz}$, which agrees with the theoretical center frequencies of radial acoustic resonance peaks. This theoretical $v_{\mathrm{GB}, m}$ value was calculated by substituting $v=5590 \mathrm{~m} / \mathrm{s}$ and $\alpha=0.624$ [11] into Eq. (1) (see Fig. 4(b)). The frequency range in which acoustic resonance peaks were observed was approximately three times broader than that of the small-core photonic crystal fibers [5]. The CFBS-BFS values of each peak agreed well with the theoretical values of each $\mathrm{R}_{0, m}$ mode [1].

Next, we measured the pump power dependence of the CFBS spectrum near the peak at $\sim 941 \mathrm{MHz}$ (corresponding to the $\mathrm{R}_{0,20}$ mode, which exhibited one of the clearest peaks in the higher frequency section). The probe power was $-5.0 \mathrm{dBm}$. The spectral peak power increased with the pump power, but the noise floor also increased (Fig. 5(a)). Therefore, here we define the SNR as the ratio between the spectral peak power and the spectral power at $961 \mathrm{MHz}$. Figure 5(b) shows the pump power dependence of the CFBS spectrum, which was normalized so that the spectral power at $961 \mathrm{MHz}$ was $0 \mathrm{~dB}$. The spectrum at the pump power of $10.0 \mathrm{dBm}$ (regarded as the noise floor of the ESA) was subtracted from all the spectra. The SNR increased with increasing pump power as shown in Fig. 5(c). The threshold 

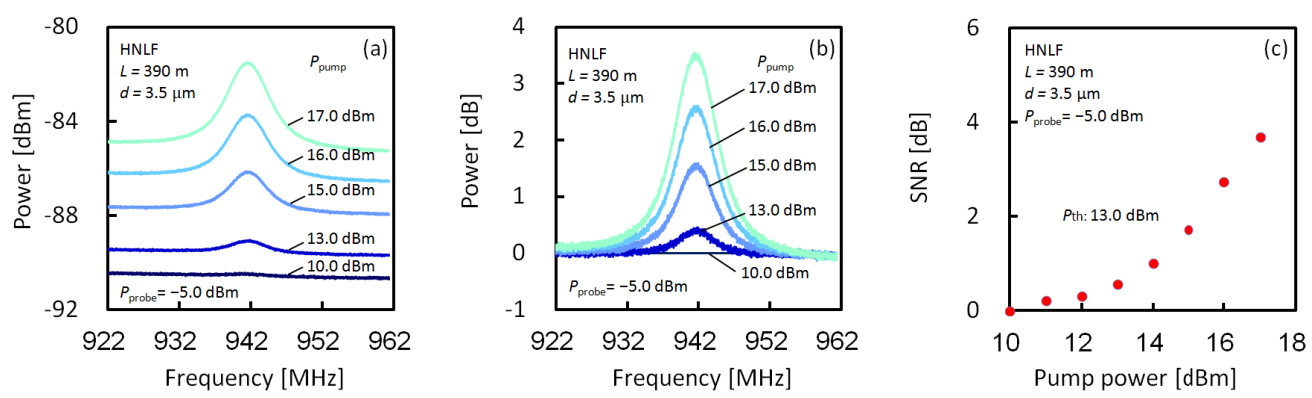

Fig. 5 (a) Pump power dependence of the CFBS spectrum around $941 \mathrm{MHz}$. (b) Pump power dependence of the CFBS spectrum normal55ized in order for the spectral power at $961 \mathrm{MHz}$ to be $0 \mathrm{~dB}$. (c) Pump power dependence of the signal-to-noise ratio (SNR) of the CFBS measurement.
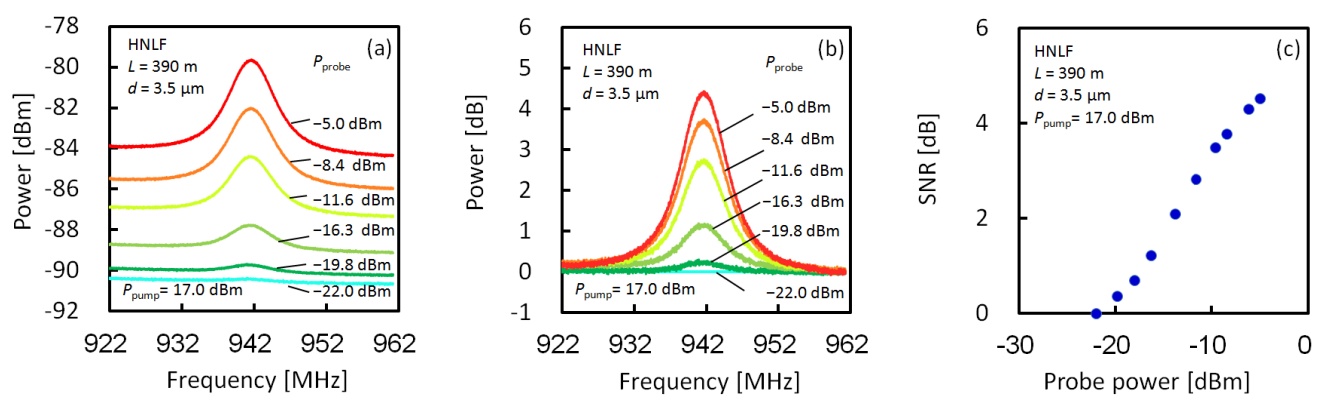

Fig. 6 (a) Probe power dependence of the CFBS spectrum around $941 \mathrm{MHz}$. (b) Probe power dependence of the CFBS spectrum normalized in order for the spectral power at $961 \mathrm{MHz}$ to be $0 \mathrm{~dB}$. (c) Probe power dependence of the SNR of the CFBS measurement for a pump power of $17.0 \mathrm{dBm}$.
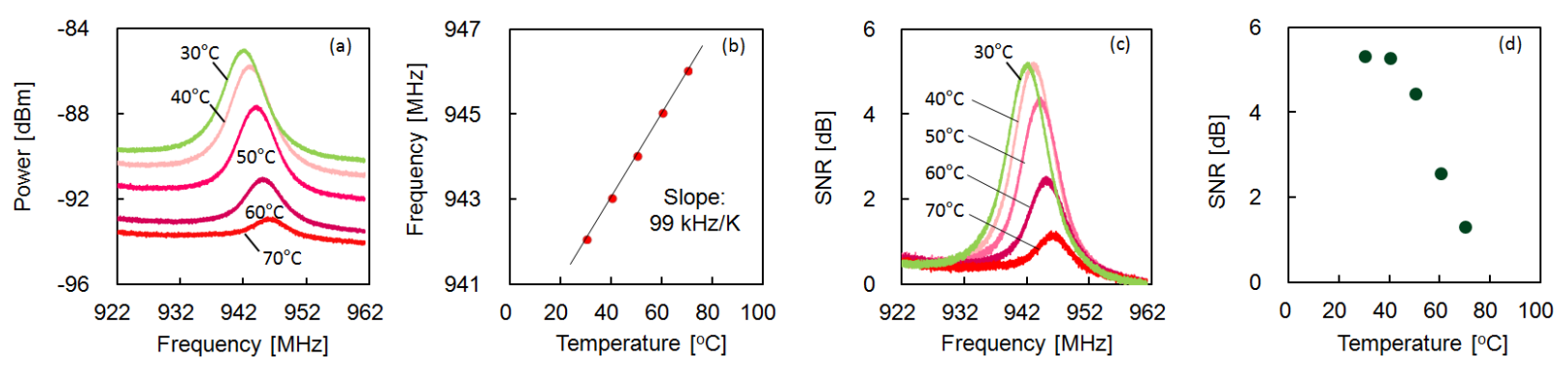

Fig. 7 (a) Temperature dependence of the CFBS spectrum around $941 \mathrm{MHz}$. (b) Temperature dependence of the center frequency shift of the spectrum. The solid line is a linear fit. (c) Temperature dependence of the CFBS spectrum normalized in order for the spectral power at $961 \mathrm{MHz}$ to be $0 \mathrm{~dB}$. (d) Temperature dependence of the SNR.

of the CFBS was $\sim 13.0 \mathrm{dBm}$, which was the almost the same as that of BSBS. Then, the SNR was limited to $4.4 \mathrm{~dB}$ from 17.0-dBm pump power by limiting the maximum power of the EDFA in this experiment.

We also measured the probe power dependence of the CFBS spectrum at $941 \mathrm{MHz}$. The pump power was $17.0 \mathrm{dBm}$. As shown in Fig. 6(a), as the probe power increased, the spectral peak power and the noise floor increased. The probe power dependence of the CFBS spectrum normalized using the spectral power at $961 \mathrm{MHz}$ is shown in Fig. 6(b). The spectrum at the probe power of $-22.0 \mathrm{dBm}$ was subtracted from all the spectra in order to suppress the influence of the noise of the ESA. The SNR became maximal at a probe power of $-5.0 \mathrm{dBm}$. The SNR dependence on the probe power is shown in Fig. 6(c). The SNR was almost linearly increased up to $-5.0 \mathrm{dBm}$. The highest SNR was measured to be $4.4 \mathrm{~dB}$ when the pump power was $17 \mathrm{dBm}$ and the probe power was $-5.0 \mathrm{dBm}$. The probe power was limited by the input power of the PD in this experiment.

Finally, we measured the temperature dependence of the CFBS spectrum. The temperature of the FUT was changed using a thermostatic chamber from $30^{\circ} \mathrm{C}$ to $70^{\circ} \mathrm{C}$. The spectrum near the peak at $\sim 941 \mathrm{MHz}$ dependence on temperature is shown in Fig. 7(a). The center frequency of the spectrum was shifted to a higher frequency with increasing temperature. The power of the spectrum decreased for the temperature range $30^{\circ} \mathrm{C}$ to $70^{\circ} \mathrm{C}$. The temperature dependence of the center frequency shift of the spectra is shown in Fig. 7(b). The dependence was linear with a coefficient of $99 \mathrm{kHz} / \mathrm{K}$, which is close to the value calculated using Eq. (3). The slight difference between the measured value and the theoretical value originates from the material properties [38]. This coefficient is clearly different from that of BSBS in the HNLF [39]. The spectra normalized in order for the power at $961 \mathrm{MHz}$ to be $0 \mathrm{~dB}$ is shown in Fig. 7(c). The SNR decreased with increasing temperature, as shown in Fig. 7(d). The SNR remained almost the same between 
$30^{\circ} \mathrm{C}$ and $40^{\circ} \mathrm{C}$, but then decreased linearly from $40^{\circ} \mathrm{C}$ to $70^{\circ} \mathrm{C}$. Although the BFS of CFBS and that of BSBS were both linearly dependent on temperature, this nonlinear SNR dependence on temperature was observed, probably because of the Lorentzian shape of the BSBS spectrum used as seed. If the center frequency of the probe light is fixed at only the first acoustic resonance frequency, the temperature measurement range is limited. For wide-range measurement of temperature, optimization of the center frequency of the probe light is required. This information will be useful for discriminating temperature and other parameters in BSBSseeded CFBS-based sensing systems in the future.

\section{Conclusion}

This paper proposed a method for generating BSBS-seeded CFBS using a counter-propagated pump-probe technique. Experimental results using the 390-m-long HNLF indicate that the CFBS had a relatively high SNR and that the center frequencies of its acoustic resonance peaks agreed with theoretical values. The Brillouin properties of the generated CFBS, such as the SNR dependence on pump power, the SNR dependence on probe power, and the BFS dependence on temperature, were then clarified. As for the SNR dependence on pump power, the threshold of CFBS was measured to be $13.0 \mathrm{dBm}$ with a $-5.0-\mathrm{dBm}$ probe light, which was almost identical to that of BSBS. In contrast, as for the SNR dependence on probe power, the SNR of the CFBS became maximal with a $17.0-\mathrm{dBm}$ pump light and a $-5.0-\mathrm{dBm}$ probe light. The temperature dependence coefficient of the CFBS-BFS values was measured to be 99 $\mathrm{kHz} / \mathrm{K}$, which was close to the theoretical value. We believe that these findings will aid the development of CFBS-based distributed acoustic-impedance sensors for underground oil layer detection, cancer detection in the human body, etc., in the near future.

\section{Acknowledgments}

This work was partly supported by JSPS KAKENHI (17H04930, 16H00902, 17J07226, 25009080, and 17K14692) and by research grants from the Noguchi Institute and the Telecommunications Advancement Foundation.

\section{References}

[1] R.M. Shelby, et al.: "Guided acoustic-wave Brillouin scattering," Phys. Rev. B 31 (1985) 5244 (DOI: 10.1103/physrevb.31.5244).

[2] A.J. Poustie: "Bandwidth and mode intensities of guided acousticwave Brillouin scattering in optical fibers," J. Opt. Soc. Am. B 10 (1993) 691 (DOI: 10.1364/josab.10.000691).

[3] N. Nishizawa, et al.: "Characteristics of guided acoustic wave Brillouin scattering in polarization maintaining fibers," Opt. Rev. 3 (1996) 29 (DOI: 10.1007/s10043-996-0029-2).

[4] T. Matsui, et al:: "Guided acoustic-wave Brillouin scattering characteristics of few-mode fiber," Appl. Opt. 54 (2015) 6093 (DOI: 10.1364/ao.54.006093).

[5] M.S. Kang, et al:: "Tightly trapped acoustic phonons in photonic crystal fibres as highly nonlinear artificial Raman oscillators," Nat. Phys. 5 (2009) 276 (DOI: 10.1038/nphys1217).

[6] J. Wang, et al.: "FSBS resonances observed in a standard highly nonlinear fiber," Opt. Express 19 (2011) 5339 (DOI: 10.1364/oe.19.005339)
[7] E.A. Kittlaus, et al.: “On-chip inter-modal Brillouin scattering," Nat. Photon. 10 (2016) 463 (DOI: 10.1038/ncomms15819).

[8] C. Wolff, et al.: "Cascaded forward Brillouin scattering to all Stokes orders,” New J. Phys. 19 (2017) 023021 (DOI: 10.1088/13672630/aa599e).

[9] N. Hayashi, et al:: "Observation of backward guidedacoustic-wave Brillouin scattering in optical fibers using pumpprobe technique," IEEE Photon. J. 8 (2016) 7100707 (DOI: 10.1109/jphot.2016.2550321).

[10] Y. London, et al.: "Electro-opto-mechanical radio-frequency oscillator driven by guided acoustic waves in standard single-mode fiber,' APL Photon. 2 (2017) 041303 (DOI: 10.1063/1.4977904).

[11] Y. Tanaka and K. Ogusu: "Temperature coefficient of sideband frequencies produced by depolarized guided acoustic-wave Brillouin scattering," IEEE Photon. Technol. Lett. 10 (1998) 1769 (DOI: 10.1109/68.730497).

[12] Y. Tanaka and K. Ogusu: "Tensile-strain coefficient of resonance frequency of depolarized guided acoustic-wave Brillouin scattering," IEEE Photon. Technol. Lett. 11 (1999) 865 (DOI: 10.1109/68.769734).

[13] M. Ohashi, et al.: "Fibre diameter estimation based on guided acoustic wave Brillouin scattering," Electron. Lett. 28 (1992) 900 (DOI: 10.1049/el:19920571).

[14] Y. Antman, et al.: "Optomechanical sensing of liquids outside standard fibers using forward stimulated Brillouin scattering," Optica 3 (2016) 510 (DOI: 10.1364/optica.3.000510).

[15] N. Hayashi, et al.: "Experimental study on depolarized GAWBS spectrum for optomechanical sensing of liquids outside standard fibers," Opt. Express 25 (2017) 2239 (DOI: 10.1364/oe.25.002239).

[16] D.M. Chow, et al.: "Frequency-domain technique to measure the inertial response of forward stimulated Brillouin scattering for acoustic impedance sensing," Proc. SPIE 10323 (2017) 1032311 (DOI: 10.1117/12.2265736).

[17] T. Horiguchi and M. Tateda: "BOTDA-nondestructive measurement of single-mode optical fiber attenuation characteristics using Brillouin interaction: theory," J. Lightw. Technol. 7 (1989) 1170 (DOI: 10.1109/50.32378).

[18] J. Urricelqui, et al.: "Phasorial differential pulse-width pair technique for long-range Brillouin optical time-domain analysis sensors," Opt. Express 22 (1989) 17403 (DOI: 10.1364/oe.22.017403).

[19] Y. Dong, et al.: "High-sensitivity distributed transverse load sensor with an elliptical-core fiber based on Brillouin dynamic gratings," Opt. Lett. 40 (2015) 5003 (DOI: 10.1364/ol.40.005003).

[20] Y. Dong, et al.: "High-spatial-resolution fast BOTDA for dynamic strain measurement based on differential double-pulse and secondorder sideband of modulation,” IEEE Photon. J. 5 (2013) 2600407 (DOI: 10.1109/jphot.2013.2267532).

[21] T. Kurashima, et al.: "Brillouin optical-fiber time domain reflectometry," IEICE Trans. Commun. E76-B (1993) 382. (DOI: 10.1364/ofc.2009.omp7)

[22] L.E.Y. Herrera, et al.: "Investigation of bend loss in single mode fibers with ultra-high-resolution photon-counting optical time domain reflectometer," Appl. Opt. 55 (2016) 1177 (DOI: 10.1364/ao.55.001177).

[23] K. Hotate and T. Hasegawa: "Measurement of Brillouin gain spectrum distribution along an optical fiber using a correlation-based technique-proposal, experiment and simulation," IEICE Trans. Electron. E83-C (2000) 405. (DOI: 10.1117/12.2302134)

[24] R.K. Yamashita, et al:: "Measurement range elongation based on temporal gating in Brillouin optical correlation domain distributed simultaneous sensing of strain and temperature," IEEE Photon. Technol. Lett. 24 (2012) 1006 (DOI: 10.1109/lpt.2012.2192725).

[25] Y. Mizuno, et al:: "Proposal of Brillouin optical correlation-domain reflectometry (BOCDR)," Opt. Express 16 (2008) 12148 (DOI: 10.1364/oe.16.012148)

[26] M.L. Palmeri, et al.: "Noninvasive evaluation of hepatic fibrosis using acoustic radiation force-based shear stiffness in patients with nonalcoholic fatty liver disease," J. Hepatol. 55 (2011) 666 (DOI: 10.1016/j.jhep.2010.12.019).

[27] Y. Kato, et al.: "Measurement of elastic wave propagation velocity near tissue surface by optical coherence tomography and laser 
Doppler velocimetry,” Jpn. J. Appl. Phys. 53 (2014) 07KF05 (DOI: 10.7567/jjap.53.07kf05).

[28] Schlumberger, Ltd.: Log Interpretation Principles/Applications (Schlumberger Educational Services, Texas, 1991). (DOI: 10.30632/pjv60n5-2019a1)

[29] S.K. Srivastava, et al.: "Surface plasmon resonance based fiber optic sensor for the detection of low water content in ethanol," Sens. Actuat. B Chem. 153 (2011) 194 (DOI: 10.1016/j.snb.2010.10.038).

[30] D. Ma, et al.: "Wireless sensor network for continuous monitoring water quality in aquaculture farm," Sens. Lett. 8 (2010) 109 (DOI: 10.1166/s1.2010.1210).

[31] H.H. Diamandi, et al.: "Opto-mechanical inter-core cross-talk in multi-core fibers," Optica 4 (2017) 289 (DOI: 10.1364/optica. 4.000289).

[32] G. Bashan, et al.: "Optomechanical time-domain reflectometry," Nat. Commun. 9 (2018) 2991 (DOI: 10.1038/s41467-018-05404-0).

[33] D.M. Chow, et al.: "Distributed forward Brillouin sensor based on local light phase recovery," Nat. Commun. 9 (2018) 2990 (DOI: 10.1038/s41467-018-05410-2).

[34] Y. Tanaka, et al.: "Guided-acoustic-wave Brillouin scattering observed backward by stimulated Brillouin scattering," Meas. Sci. Technol. 15 (2004) 1458 (DOI: 10.1088/0957-0233/15/8/004).

[35] N. Hayashi, et al.: "Fiber-optic cascaded forward Brillouin scattering seeded by backward SBS," 2018 Conference on Lasers and Electro-Optics (CLEO) (2018) SM3K.2 (DOI: 10.1364/cleo_si.2018.sm3k.2).

[36] Y. Antman, et al.: "Scanning-free characterization of temperature dependence of forward stimulated Brillouin scattering resonances," Proc. SPIE 9634 (2015) 96345C (DOI: 10.1117/12.2195097).

[37] G.P. Agrawal: Nonlinear Fiber Optics (Academic Press, California, 1995) (DOI: 10.1016/b978-0-12-045142-5.50018-0).

[38] N. Hayashi, et al:: "Temperature coefficient of sideband frequency produced by polarized guided acoustic-wave Brillouin scattering in highly nonlinear fibers," Appl. Phys. Express 10 (2017) 092501 (DOI: 10.7567/apex.10.092501).

[39] M.R. Lorenzen, et al:: "Suppression of Brillouin scattering in fibre-optical parametric amplifier by applying temperature control and phase modulation," Electron. Lett. 45 (2009) 125 (DOI: 10.1049/el:20092757). 